DIAGNOSTICS

\title{
Use of PCR in the diagnosis of early syphilis in the United Kingdom
}

\author{
H M Palmer, S P Higgins, A J Herring, M A Kingston
}

Sex Transm Infect 2003;79:479-483

See end of article for authors' affiliations

.....................

Correspondence to: Helen M Palmer, Scottish Neisseria gonorrhoeae Reference Laboratory, Department of Medical Microbiology, Royal Infirmary of Edinburgh, Little France, Edinburgh, EH16 4SA, UK; helen.palmer@ luht.scot.nhs.uk

Accepted for publication 7 July 2003

\begin{abstract}
Objectives: To evaluate a Treponema pallidum polymerase chain reaction (PCR) test in the laboratory diagnosis of early syphilis in the United Kingdom.

Subjects and setting: Men and women attending genitourinary medicine clinics in England.

Methods: A trial PCR service was offered for the analysis of swabs of ano-genital or oral ulcers suspected to be syphilitic in origin. Clinical details, results of treponemal serology, and other relevant laboratory tests carried out by the sending laboratories were obtained retrospectively by questionnaire.

Results: Data from 98 patients, representing 100 episodes of ulceration, were analysed. The majority of patients (70) attended clinics in the Greater Manchester area. Eighty six patients were male and 58 were men who have sex with men (MSM), of whom 24 were HIV positive. PCR results agreed with the clinical diagnosis for 95 patients; samples from 26 patients were PCR positive and serologically diagnosed as primary (18) or secondary (8) syphilis, whereas 70 patients had PCR negative samples and were not diagnosed as having active syphilis. These data include two HIV positive patients who were PCR positive 12 and 21 days before their treponemal seroconversion. One positive PCR result was not supported by positive treponemal serology (this patient coincidentally received a 10 day course of co-amoxiclav 1 week after sampling). Three patients had negative PCR results but positive syphilis serology. The sensitivity, specificity, positive and negative predictive value for primary syphilis were 94.7\%,98.6\%, 94.7\%, and $98.6 \%$, respectively, and for secondary syphilis these were $80.0 \%, 98.6 \%, 88.9 \%$, and $97.2 \%$, respectively.

Conclusion: PCR is a sensitive and specific test for $T$ pallidum, and an important adjunct to dark ground microscopy and treponemal serology in diagnosing infectious syphilis in the United Kingdom.
\end{abstract}

A fter falling to an all time low in the early 1990s, the prevalence of infectious syphilis (primary and secondary stages) in the United Kingdom rose substantially after 1996. This increase was largely the result of localised outbreaks in Bristol, ${ }^{1}$ Brighton, ${ }^{2}$ Manchester, ${ }^{3}$ and Cambridgeshire. ${ }^{4}$ There was also an outbreak in Dublin, Ireland. ${ }^{5}$ Some outbreaks involved a substantial number of HIV positive men who have sex with men (MSM). The re-emergence of syphilis in the HIV era is a particular cause for concern, as genital ulcer disease is known to facilitate the spread of HIV infection. ${ }^{6}$ The public health response to these syphilis outbreaks has included health promotion campaigns, community based screening projects, and increased access to departments of genitourinary medicine. $^{7}$

Primary and secondary syphilis are diagnosed on the basis of symptoms and clinical signs, with confirmation by treponemal serology. Diagnosing syphilis in HIV co-infected patients can be difficult, particularly in individuals with low CD4 lymphocyte counts, because patients may present with atypical symptoms, such as herpetiform ulceration. ${ }^{8}$ Serological screening tests for syphilis include cardiolipin based tests (Venereal Disease Research Laboratory (VDRL) test and rapid plasma reagin (RPR) test) and treponemal specific antibody tests (Treponema pallidum haemagglutination assay (TPHA), T pallidum particle agglutination assay (TPPA), fluorescent treponemal antibody test (FTA), or treponemal enzyme immunoassay (EIA), which detect IgG and/or IgM). ${ }^{9}$ The sensitivity of these tests varies for primary syphilis: VDRL/RPR (70-80\%), TPHA/TPPA (70-80\%), treponemal EIA (85-90\%), and FTA-abs (85-90\%). ${ }^{10}$ A serological response to the presence of $T$ pallidum usually takes between 1-4 weeks to develop, and is normally present by the time the primary chancre is seen, with the earliest serological detection being achieved by IgM specific EIAs. ${ }^{11}$ However, serological screening tests may fail to detect up to $30 \%$ of primary syphilis.

Syphilitic ulceration, most commonly seen on the anogenital skin or in the mouth, offers an opportunity for direct detection of $T$ pallidum early in infection. In the United Kingdom, the only direct detection method used is dark ground microscopy (DGM), which has demonstrated a sensitivity of $79-97 \%$ and a specificity of $77-100 \%$ in previous studies. ${ }^{12-15}$ However, DGM requires levels of skill and experience that are no longer common in the United Kingdom, and this test is unsuitable for specimens from the mouth or rectum because non-pathogenic spirochaetes may exist at these sites. Direct fluorescent antibody (DFA) testing is an alternative to dark ground microscopy, ${ }^{13}$ but this is not available in the United Kingdom. Polymerase chain reaction (PCR) offers an attractive option for the direct detection of $T$ pallidum and several protocols have already been described. ${ }^{15-21}$ The assays are pathogenic treponeme specific: $95-97 \%,{ }^{15} 21$ so oral and rectal specimens may be tested; and sensitive: $91-95 \%,{ }^{151}$ with a reported limit of detection as low as 1-65 organisms..$^{15-19} 21$

In response to the increase in infectious syphilis in the United Kingdom, the Genitourinary Infections Reference Laboratory (GUIRL) offered PCR testing for syphilis on a trial basis. The PCR developed by Orle et $a l^{15}$ was used because it has been widely used in clinical studies ${ }^{22-28}$ and, in its complete form, allows the detection of three pathogens that cause genital ulcers (Haemophilus ducreyi, herpes simplex virus (HSV), and T pallidum. In this paper we report the results obtained with the PCR test and compare them with the diagnoses given by the sending clinicians, which were made 
on the basis of their clinical information and laboratory test results.

\section{METHODS}

\section{Transport and storage of samples}

Swabs of ano-genital or oral ulcers that were suspected to be syphilitic were requested between March 2000 and September 2001. These were sent either in dry sterile containers or placed in vials containing $1-1.5 \mathrm{ml}$ transport medium-for example, viral or chlamydia transport medium. Samples were posted to the GUIRL and refrigerated $\left(4^{\circ} \mathrm{C}\right)$ upon receipt.

\section{Sample preparation}

Batches of samples were processed on a weekly basis. Dry swabs were hydrated with $1 \mathrm{ml}$ sterile saline solution for 1-5 minutes. All swabs were vortexed briefly before the saline solution or transport medium was transferred to sterile $1.5 \mathrm{ml}$ tubes and centrifuged $(13000 \mathrm{~g})$ for 1 minute. The top $0.8-1.3 \mathrm{ml}$ of supernatant was removed and discarded leaving the remaining $200 \mu \mathrm{l}$ supernatant and any cellular deposit for DNA extraction. A negative control sample $(1 \mathrm{ml}$ sterile saline) was included in every batch of samples. DNA extraction was carried out using the QIAamp DNA Blood mini kit (Qiagen Ltd, Crawley, West Sussex, UK) using the blood and body fluid spin protocol. DNA was eluted in $150 \mu \mathrm{l}$ sterile distilled water, and a $25 \mu \mathrm{l}$ aliquot was used for the PCR assay.

\section{PCR and detection}

The primers and thermal cycling conditions from the genital ulcer disease multiplex PCR were used. ${ }^{15}$ This amplifies a $260 \mathrm{bp}$ region of the $47 \mathrm{kDa}$ integral membrane lipoprotein gene using the following primers: KO3A 5' GAAGTTTGTCCCAGTTGCGGTT and KO4 5'CAGAGCCATCAGCCCTTTTCA. Each reaction contained $0.5 \mu \mathrm{M}$ primers KO3A and KO4, $1 \times$ PCR buffer $(50 \mathrm{mM}$ $\mathrm{KCl}, 20 \mathrm{mM}$ TRIS-HCl pH 8.4), $1.5 \mathrm{mM} \mathrm{MgCl}_{2}, 0.2 \mathrm{mM}$ dNTPs, and 1.25 units of Platinum Taq DNA polymerase (Invitrogen Life Technologies, Paisley, UK). Included in each PCR run were a negative control sample $(25 \mu$ distilled water), a positive control sample (distilled water containing $100 \mathrm{pg}$ T pallidum DNA), and an inhibition control for each sample $(25 \mu$ l sample spiked with $100 \mathrm{pg} T$ pallidum DNA). Amplification was carried out on a Perkin Elmer 9700 using the 9600 ramp setting and the published thermal cycling conditions, which were as follows: $95^{\circ} \mathrm{C}$ for 2 minutes, then 35 cycles of $95^{\circ} \mathrm{C}$ for 20 seconds, $62^{\circ} \mathrm{C}$ for 20 seconds, and $72^{\circ} \mathrm{C}$ for 20 seconds. PCR products were analysed by electrophoresis using a $2 \%$ agarose gel.

\section{Control DNA}

Using standard methods, ${ }^{29}$ genomic DNA was extracted from purified $T$ pallidum Nicols strain (Newmarket Laboratories Ltd, Newmarket, UK) and quantified by ultraviolet spectrometry. A serial dilution of purified T pallidum DNA was used to determine the detection limit of the PCR.

\section{Clinical details and laboratory test results}

Demographic and clinical details and relevant laboratory test results were obtained retrospectively by questionnaire. Data requested included the date and results from DGM (if performed) and serological tests, details of any antibiotic treatment before obtaining the swab sample, the HIV status of the individual and their sexual orientation. Clinicians responding to the questionnaire were asked to classify their diagnosis of each case as primary syphilis, secondary syphilis, or "not syphilis" according to all the available test results and to indicate if an alternative laboratory diagnosis was made-for example, an HSV infection. The particular laboratory tests used varied according to local diagnostic practices. The serological tests used included RPR, VDRL, TPHA, TPPA, an EIA detecting IgM and IgG, and an EIA detecting IgM only.

\section{RESULTS}

\section{Demography}

Samples were submitted from 117 patients, 98 of which are analysed below (see table 1). Data from 14 patients were incomplete (no questionnaire was returned) and excluded from this study. Samples from three patients were not from ulcers and were excluded from further analysis; two were swabs of skin rashes (on the foot and chest) and one was a swab of a sacral abscess (although these sites are suitable for sampling, a scraping rather than a swab is required to ensure an adequate sample is provided). After analysis of the serological data, samples from a further two patients were excluded: one patient was diagnosed clinically as having primary syphilis, with no confirmatory serology; the second was diagnosed with early latent syphilis and therefore by definition any ulcer sampled (in this case a mouth ulcer) would not be caused by T pallidum.

The majority of patients had attended GUM clinics in the Greater Manchester area (70) with others attending clinics in London (19), Poole (five), Bristol (two), Dublin (one), and Worcester (one). Most patients (86) were male and, of these, a majority were MSM (58). Of the MSM, 24 were known to be HIV positive, with a mean CD4 lymphocyte count of $353 \times 10^{6} / 1$ (range 65-641, normal range 500-1500; the count for two of these patients was unknown). Nine were receiving highly active antiretroviral treatment.

\section{PCR testing}

Swab specimens were obtained from the following sites: penis (47); oral cavity, tongue and lips (25); anus (18); vulva (12); rectum (three); and no site was specified for eight ulcer swabs. Duplicate samples (either two swabs of different ulcers or duplicate swabs taken on sequential clinic visits) were submitted from 13 patients. Three swab samples were submitted from one patient. Samples from these 14 patients represented 16 episodes of ulceration (two patients presented with two separate infections during the 19 month period of this study). One sample per episode of ulceration was included in the analysis. For six patients both samples were positive by PCR and for five patients both samples were negative by PCR

One patient diagnosed with primary syphilis had a PCR positive sample at presentation and a PCR negative sample a week later, following treatment with procaine penicillin; these samples represent a single episode of infection and only the first sample was included in the analysis.

Another patient, diagnosed with primary syphilis, had a PCR positive penile ulcer sample at presentation and was treated with a 17 day course of procaine penicillin; 2 days after completion of treatment, he presented with a peri-anal ulcer that was PCR negative but HSV culture positive. These two PCR samples represent two different episodes of ulceration (although it is not known whether the HSV infection was newly acquired or recurrent) and both were included in the analysis.

One patient, presenting with a penile ulcer, had negative serology and a negative PCR test on the first visit and was diagnosed as "not syphilis," but 5 months later presented with penile and oral ulcers, both PCR positive, and had positive treponemal serology consistent with secondary syphilis. These three samples represent two episodes of ulceration; the first sample and only one sample from the second visit were included in the analysis. It is possible that 


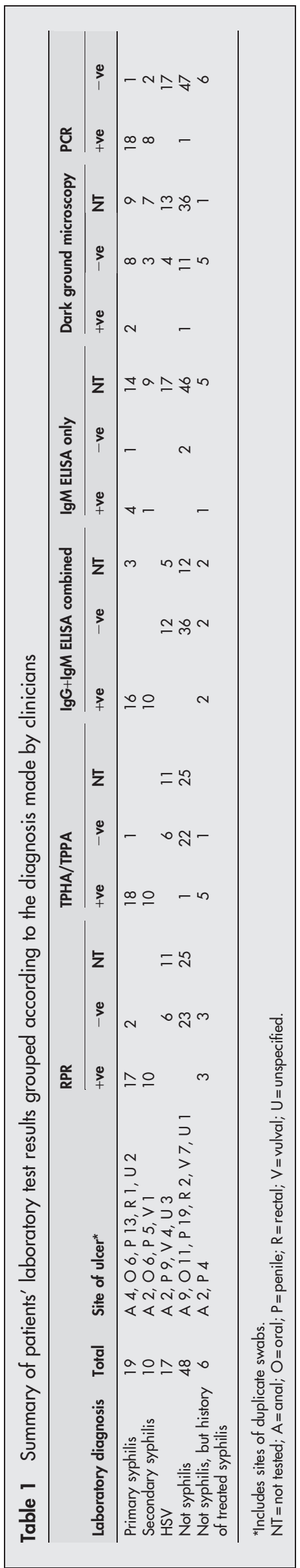

the first presentation was primary syphilis before seroconversion, although this cannot be proved.

\section{PCR detection limit}

The detection limit of the PCR was determined as $1 \mathrm{pg}$ $T$ pallidum DNA representing approximately 800 genome copies. All negative DNA extraction and PCR controls included in each batch of samples were negative and all positive PCR controls were positive. Inhibition tests of all samples revealed that there was no inhibition of the PCR reaction.

\section{Clinical diagnosis and laboratory test results}

Table 1 summarises details of serological tests, DGM and PCR, with patients grouped according to the diagnosis given by the sending clinician on the basis of clinical details and laboratory test results. Twenty nine cases of syphilis were identified by serology; 19 were defined as primary and 10 as secondary syphilis. Only five of 19 primary cases had a serological test to detect $\operatorname{Ig} M$ alone; four were $\operatorname{Ig} M$ positive and the fifth, who had no detectable anti-treponemal IgM, was a re-infection of a case previously identified as secondary syphilis in 1992 and subsequently treated. DGM was carried out on specimens from 13 patients with syphilis and was positive for two of the primary cases. PCR was carried out on specimens from all 30 cases of syphilis and was positive in 26 cases (18 primary and eight secondary cases). Two HIV positive patients (one with a CD4 lymphocyte count of 350, the count for the second was unknown) tested PCR positive 12 and 21 days, respectively, before syphilis seroconversion.

There were 71 patients who were diagnosed as not having syphilis, including 17 cases for whom a laboratory confirmed diagnosis of HSV was given and six with a previous history of treated syphilis. DGM was carried out on specimens from 21 of 71 patients, 20 of which were negative. One specimen, an anal swab, was positive, but the serology for this patient (RPR, TPHA, and an IgG+IgM EIA) was negative on two occasions, hence the clinician's classification of this case as "not syphilis" (the PCR result for this patient was negative). It is possible that the DGM positive anal ulcer represents a case of syphilis before seroconversion although this cannot be verified. PCR was negative for samples from 70 of the 71 cases defined as "not syphilis."

\section{Discrepancies between serological and PCR results}

One case of primary syphilis (two samples from this patient, one penile and one mouth ulcer swab) and two cases of secondary syphilis (two penile swabs and one mouth swab) were PCR negative.

One case, diagnosed as "not syphilis" on the basis of consistently negative treponemal serology (RPR, TPHA, and EIAs for IgM only and IgM+IgG combined were negative on three occasions) had a PCR positive penile swab. Only two other samples were processed alongside this sample in the laboratory, neither of which were PCR positive. This patient was HIV positive with a CD4 lymphocyte count of 180 (normal range 500-1500) and, coincidentally, had received a course of co-amoxiclav 6 days after the PCR positive swab was taken.

\section{Performance of the PCR test}

The sensitivity, specificity, positive and negative predictive values for the PCR were calculated as $94.7 \%, 98.6 \%, 94.7 \%$, and $98.6 \%$, respectively, for primary syphilis and $80 \%, 98.6 \%$, $88.9 \%$, and $97.2 \%$, respectively, for secondary syphilis.

\section{DISCUSSION}

The epidemiology of syphilis in the United Kingdom has undergone significant changes recently. ${ }^{30}$ Localised outbreaks 
have resulted in many patients presenting with ulcerative lesions suitable for direct detection of $T$ pallidum by PCR, in sites where DGM cannot be used (mainly oral lesions). In response to the changing epidemiology of syphilis, we have offered a $T$ pallidum specific PCR assay as a trial diagnostic service. Over the 19 month period of this study, swabs from 117 patients were submitted for syphilis PCR, indicating that a demand for the service existed, at least in some areas where syphilis was prevalent (principally Manchester and London).

Data presented in table 1 demonstrate that for the majority of cases the PCR results correlate well with the serology obtained; 18/19 primary and 8/10 secondary cases of syphilis were detected by PCR. Two HIV positive patients were positive by PCR more than 1 week before treponemal seroconversion.

The PCR successfully detected $T$ pallidum from ulcers present during primary or secondary syphilis from oral, genital, and anal sites. These data served to confirm positive cases or, on occasion, to pre-empt the serological results. Negative PCR results were also of value, allowing the prompt elimination of syphilis from the diagnosis in many instances. HSV infection was a frequent diagnosis among those cases that were identified as "not syphilis," which suggests that a PCR offering a differential diagnosis between $T$ pallidum and HSV would be a valuable tool in regions of the United Kingdom where syphilis outbreaks are ongoing. This is possible if the multiplex ulcer $\mathrm{PCR}^{15}$ is used in its entirety, and it would also detect the occasional $H$ ducreyi infection.

There were four discrepancies between diagnoses given and the PCR results. Three patients with serologically proved syphilis gave false negative PCR results: these may represent sampling ulcers of non-treponemal origin in patients with coincident positive treponemal serology or simply reflect the limited sensitivity of the PCR in the simplified format used here. Ulcer specimens typically contain $22 \times 10^{3}-5.7 \times 10^{6}$ organisms $^{31}$; hence a detection limit of 1 pg DNA (equivalent to approximately 800 organisms) should have been adequate. However, the number of organisms present in resolving ulcers may be small and sampling technique may vary. A PCR with a lower limit of detection could be achieved either by using the enzyme linked oligonucleotide sorbent assay to detect the PCR product (as advocated in the original paper ${ }^{15}$ ) or by converting the PCR to one of the real time PCR platforms (Light cycler or Taqman).

The final discrepant result was a false-positive PCR result in a patient with consistently negative syphilis serology. Unfortunately, several DNA samples (including this one) were lost to the study and so it was not possible to carry out discrepant analysis using an alternative $T$ pallidum PCR. One possibility for this discrepancy is that prompt antibiotic treatment (coincidental) coupled with the patient's immune dysfunction may have blunted a serological response to T pallidum. Much of the syphilis literature relies on diagnosis by serology, hence serologically negative cases of syphilis in patients with immune dysfunction may be underestimated.

DGM was used infrequently, in only 34 of 84 suitable samples. This finding is not surprising, as generations of UK trained genitourinary physicians have seen little if any early syphilis and have not acquired the expertise necessary for diagnosis using DGM. There is a dominance of samples from oral and anal sites in outbreaks of syphilis that occur within the "gay" community (as in Manchester and Brighton). Although DGM can be carried out on anal ulcers, it is not advised for oral ulcers because these usually harbour other spirochaetes.

The lack of use of specific anti-treponemal IgM testing in primary syphilis (used in only five of 19 cases) is surprising as this test has a sensitivity in excess of $80 \%{ }^{11}$ This finding also suggests lack of compliance with UK guidelines for the serological diagnosis of syphilis, which recommends specific IgM testing in suspected primary infection. ${ }^{32} 33$

Diagnostic methods that can aid rapid identification of the disease can, by expediting treatment, reduce onward transmission. A PCR test for syphilis would offer a direct detection method that is suitable for ulcer specimens from all body sites, including the mouth and rectum. PCR may provide earlier diagnosis for those patients who present with an ulcer in the serological "window period," for patients who refuse venepuncture (one in this study), and for those who fail to attend for sequential serological analysis. A diagnostic PCR service for syphilis could make a valuable contribution to what can be a difficult diagnostic process. Moreover, the acquisition of treponemal DNA from PCR positive samples could provide a valuable resource for molecular epidemiology. ${ }^{34}$ This could facilitate epidemiological investigation of syphilis, a re-emerging infection in the United Kingdom.

\section{ACKNOWLEDGEMENTS}

The authors wish to thank all those members of staff in GUM clinics and microbiology departments who were involved in the provision of swab samples for PCR and the completion of retrospective questionnaires. The authors are grateful to Dr H Young for useful discussion during the preparation of the manuscript.

\section{CONTRIBUTORS}

This work was initiated by HMP and AJH; PCR assays were carried out by HMP; SPH and MK were major providers of clinical material and details; preparation of the manuscript was carried out by HMP, $\mathrm{SPH}, \mathrm{AJH}$, and $\mathrm{MK}$.

\section{Authors' affiliations}

H M Palmer, A J Herring, Genitourinary Infections Reference Laboratory, Public Health Laboratory, Bristol Royal Infirmary, Bristol BS2 8HW, UK

S P Higgins, M A Kingston, Department of Genitourinary Medicine, North Manchester General Hospital, Delaunays Road, Crumpsall, Manchester M8 5RB, UK

\section{REFERENCES}

1 Battu VR, Horner PJ, Taylor PK, et al. Locally acquired heterosexual outbreak of syphilis in Bristol. Lancet 1997;350:1100-1.

2 Poulton M, Dean GL, Williams DI, et al. Surfing with spirochaetes: an ongoing syphilis outbreak in Brighton. Sex Transm Infect 2001;77:319-21.

3 CDSC. Increased transmission of syphilis in Manchester. Commun Dis Rep CDR Wkly 2000;10:89.

4 CDSC. Outbreak of heterosexually acquired syphilis in Cambridgeshire. Commun Dis Rep Wkly 2000;45:401-4.

5 Hopkins S, Lyons F, Mulcahy F, et al. The great pretender returns to Dublin, Ireland. Sex Transm Infect 2001;77:316-8.

6 Fleming DT, Wasserheit JN. From epidemiological synergy to public health policy and practice: the contribution of other sexually transmitted diseases to sexual transmission of HIV infection. Sex Transm Infect 1999;75:3-17.

7 Clarke P, Cook PA, Syed Q, et al. Re-emerging syphilis in the North West: lessons from the Manchester outbreak 2001. Liverpool: John Moores University, 40pp.

8 Rompalo AM, Lawlor J, Seaman $P$, et al. Modification of syphilitic genital ulcer manifestations by coexistent HIV infection. Sex Transm Dis 2001;28:448-54.

9 Wicher K, Horowitz HW, Wicher V. Laboratory methods of diagnosis of syphilis for the beginning of the third millennium. Microbes Infect 1999; 12:1035-49.

10 Young $H$, McMillan A. Syphilis and the endemic treponematoses. In: McMillan A, Young H, Ogilvie MM, Scott GR, eds. Clinical practice in sexually transmissible infections. China: Saunders, 2002

11 Schmidt BL, Edjlalipour M, Luger A. Comparative evaluation of nine different enzyme-linked immunosorbent assays for determination of antibodies against Treponema pallidum in patients with primary syphilis. J Clin Microbiol 2000;38: 1279-82.

12 Hook EW, Roddy RE, Lukehart SA, et al. Detection of Treponema pallidum in lesion exuadate with a pathogen specific antibody. J Clin Microbiol 1985:22:241-4.

13 Romanowski B, Forsey E, Prasad E, et al. Detection of Treponema pallidum by a fluorescent monoclonal antibody test. Sex Transm Dis 1987;14:156-9.

14 Cummings MC, Lukehart SA, Marra C, et al. Comparison of methods for the detection of Treponema pallidum in lesions of early syphilis. Sex Transm Dis 1996:23:366-9. 
15 Orle KA Gates CA, Martin DH et al Simultaneous PCR detection of Haemophilus ducreyi, Treponema pallidum and herpes simplex virus types and 2 from genital ulcers. J Clin Microbiol 1996;34:49-54.

16 Hay PE, Clarke JR, Strugnell RA, et al. Use of the polymerase chain reaction to detect DNA sequences specific to pathogenic treponemes in cerebrospinal fluid. FEMS Microbiol Lett 1990;68:233-8.

17 Burstain JM, Gimprel E, Lukehart SA, et al. Sensitive detection of Treponema pallidum by using the polymerase chain reaction. J Clin Microbiol 1991;29.62-9.

18 Noordhoek GT, Wolters EC, De Jonge MEJ, et al. Detection by polymerase chain reaction of Treponema pallidum DNA in cerebrospinal fluid from neurosyphilis patients before and after antibiotic treatment. J Clin Microbiol 1991;29:1976-84.

19 Centurion-Lara A, Castro C, Shaffer JM, et al. Detection of Treponema pallidum by a sensitive reverse transcriptase PCR. J Clin Microbiol 1997;35:1348-52.

20 Centurion-Lara A, Castro C, Castillo R, et al. The flanking region sequences of the 15-kDa lipoprotein gene differentiate pathogenic treponemes. J Infect Dis 1998;177:1036-40.

21 Liu H, Rodes B, Chen C-Y, et al. New tests for syphilis: rational design of a PCR method for detection of Treponema pallidum in clinical specimens using unique regions of the DNA polymerase I gene. J Clin Microbiol 2001;39:1941-6.

22 Mertz KJ, Weiss JB, Levine WC, et al. An investigation of genital ulcers in Jackson, Mississippi, with use of a multiplex polymerase chain reaction assay: high prevalence of chancroid and human immunodeficiency virus infection. $J$ Infect Dis 1998;178:1060-6.

23 Behets FM, Andriamiadana J, Randrianasolo D, et al. Chancroid, primary syphilis, genital herpes, and lymphogranuloma venereum in Antananarivo, Madagascar. J Infect Dis 1999:180:1382-5.

24 Behets FM-T, Brathwaite AR, Hylton-Kong T, et al. Genital ulcers: etiology, clinical diagnosis, and associated human immunodeficiency virus infection in Kingston, Jamaica. Clin Infect Dis 1999;28:1086-90.
25 Risbud A, Chan-Tack K, Gadkari D, et al. The etiology of genital ulcer disease by multiplex polymerase chain reaction and relationship to HIV infection among patients attending sexually transmitted disease clinics in Pune, India. Sex Transm Dis 1999;26:55-62.

26 Morse SA, Trees DL, Htun Y, et al. Comparison of clinical diagnosis and standard laboratory and molecular methods for the diagnosis of genital ulcer disease in Lesotho: association with human immunodeficiency virus infection. $J$ Infect Dis 1997; 175:583-9.

27 Beyer C, Jitwatcharanan K, Natpratan C, et al. Molecular methods for the diagnosis of genital ulcer disease in a sexually transmitted disease clinic population in Northern Thailand: predominance of herpes simplex infection. $J$ Infect Dis 1998;178:243-6.

28 Mertz KJ, Trees D, Levine WC, et al. Etiology of genital ulcers and prevalence of human immunodeficiency virus co-infection in 10 US cities. J Infect Dis 1998;178: 1795-8.

29 Ausubel FM, Brent R, Kingstown RE, et al. Preparation of genomic DNA from bacteria. In: Current protocols in molecular biology. New York: John Wiley \& Sons, 1989.

30 Fenton KA, Nicholl A, Kinghorn G. Resurgence of syphilis in England: time for more radical and nationally coordinated approaches. Sex Transm Infect $2001 ; 77: 309-10$

31 Liu H, McCaustland K, Holloway B. Evaluating the concentration of Treponema pallidum in blood and body fluid using a semi-quantitative PCR method. Int J STD AIDS 2001;12(Suppl 2):142-3.

32 Egglestone SI, Turner AJL. For the PHLS Syphilis Serology Working Group Serological diagnosis of syphilis. Commun Dis Public Health 2000;3:158-62.

33 Goh B. Management of early syphilis. (www.mssvd.org.uk/PDF/CEG2001/ early\$final0502.PDF).

34 Pillay A, Lui $\mathrm{H}$, Chen $\mathrm{CY}$, et al. Molecular subtyping of Treponema pallidum subspecies pallidum. Sex Transm Dis 1998;25:408-14.

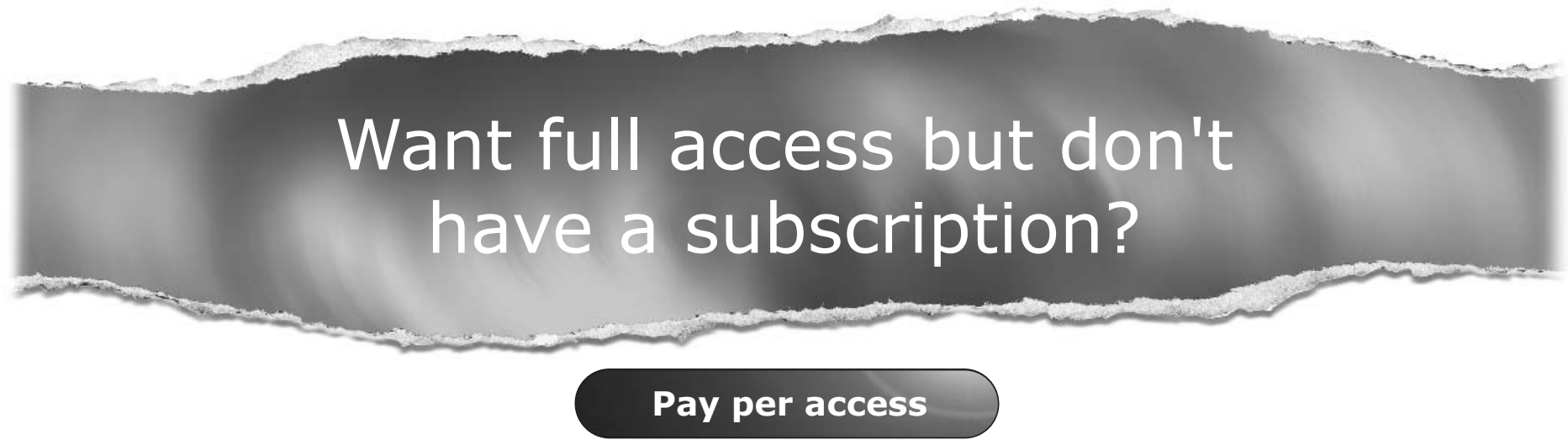

For just US\$25 you can have instant access to the whole website for 30 days. During this time you will be able to access the full text for all issues (including supplements) available. You will also be able to download and print any relevant pdf files for personal use, and take advantage of all the special features Sexually Transmitted Infections online has to offer.

\section{www.stijournal.com}

